\title{
Efficacy of Physical Activity in Prevention of Muscle Weakness in Patients With Chronic Liver Disease
}

\author{
Kazuki Ohashia, f, Toru Ishikawa ${ }^{\text {b }}$, Asami Hoshiic, Tamaki Hokaric, Mitsuyuki Suzuki ${ }^{\text {, }}$, \\ Yuta Mitobe ${ }^{\mathrm{e}}$, Etsuko Yamada ${ }^{\mathrm{f}}$, Hansani Madushika Abeywickrama ${ }^{\mathrm{f}}$, Yuko Kikuchif, \\ Hirohito Noguchig, Hiroshi Hirosawah, Yu Koyama, ${ }^{\text {f, }}$
}

\begin{abstract}
Background: Sarcopenia is a prognostic factor for patients with liver cirrhosis and hepatocellular carcinoma, and it affects the onset of hepatic encephalopathy. Therefore, the prevention of sarcopenia contributes to the improvement of the prognosis of patients with chronic liver disease (CLD). We focused on changes of hand grip strength (HGS), one of the indicators of sarcopenia. However, there are little data investigating the impact of physical activity (PA) on HGS in patients with CLD. This study aimed to clarify whether PA contributes to the prevention of muscle weakness in patients with CLD.
\end{abstract}

Methods: This was a prospective observational study. We examined the effect of PA on changes in HGS from the baseline to the endpoint in each group. Metabolic equivalents-hour/week (METs-h/w) was used to evaluate PA. In total, 183 outpatients with CLD were analyzed. We divided participants into four groups (low PA in younger patients $(n=20)$, high PA in younger patients $(n=33)$, low PA in elderly patients $(n=47)$, and high PA in elderly patients $(n=83))$.

Results: Fifty-eight percent of patients were men, and the median (interquartile range) age was $69.0(63.0,75.0)$ years. The most common etiology of liver disease was hepatitis C (38\%). The frequency of living alone and low exercise habit was significantly high, and sarcopenia was more obvious in elderly patients with low PA than in those with

Manuscript submitted September 2, 2019, accepted September 23, 2019

aDepartment of Nursing, Sapporo University of Health Sciences, Hokkaido, Japan

bepartment of Gastroenterology and Hepatology, Saiseikai Niigata Hospital, Niigata, Japan

'Department of Medical Radiology, Saiseikai Niigata Hospital, Niigata, Japan dDepartment of Pharmacology, Saiseikai Niigata Hospital, Niigata, Japan

e Department of Nursing, Teikyo University, Faculty of Medical Technology, Tokyo, Japan

fDepartment of Nursing, Graduate School of Health Sciences, Niigata University, Niigata, Japan

gDepartment of Nursing, Saiseikai Niigata Hospital, Niigata, Japan

hDepartment of Clinical Engineering, Saiseikai Niigata Hospital, Niigata, Japan

${ }^{\mathrm{i}}$ Corresponding Author: Yu Koyama, Department of Nursing, Graduate School of Health Sciences, Niigata University, 2-746 Asahimachi, Niigata 951-8518, Japan. Email: yukmy@clg.niigata-u.ac.jp

doi: https://doi.org/10.14740/jocmr3978 high PA. Additionally, the elderly with low PA showed significantly reduced HGS compared to that of the elderly with high PA (-1.00 (-2.27, $0.55) \mathrm{kg}$ vs. $0.10(-1.40,1.10) \mathrm{kg}, \mathrm{P}<0.05)$. However, changes in HGS in younger patients were not significant $(-0.02(1.83,1.47) \mathrm{kg}$ vs. 0.25 $(-2.45,2.05) \mathrm{kg}, \mathrm{P}=0.96)$. Logistic regression analyses identified PA as the independent factor for prevention of decrease in HGS (odds ratio: $1.91,95 \%$ confidence interval: $1.00-3.62, \mathrm{P}=0.049$ ).

Conclusions: Young patients with low PA were characterized by a long sedentary time; however, there was no loss of HGS. In contrast, elderly patients with CLD and low PA had significantly reduced HGS compared to that in elderly patients with CLD and high PA.

Keywords: Sarcopenia; Chronic liver disease; Physical activity; Skeletal muscle mass

\section{Introduction}

Sarcopenia is defined as a combination of low muscle mass and reduced muscle strength or impaired physical performance [1]. It is caused by malnutrition or low physical activity (PA) and a variety of clinical conditions, such as inflammatory disease, malignancy, and endocrine disease [2,3]. Recent studies have revealed that sarcopenia is a prognostic factor for patients with liver cirrhosis (LC) and hepatocellular carcinoma (HCC), and it affects the onset of hepatic encephalopathy [4-8]. Therefore, prevention of sarcopenia may contribute to the improvement of the prognosis of patients with chronic liver disease (CLD). Recently, the Society on Sarcopenia, Cachexia and Wasting Disorders recommended performing exercise thrice weekly (aerobic and resistance exercise) and consuming sufficient energy and protein and supplementing with leucine and vitamin D $[9,10]$.

Previous studies have reported improvements in physical function and quality of life in patients with LC upon administration of branched-chain amino acids (BCAAs) and training by experts [11]. However, as many of them require training according to experts, it is difficult for patients to do training on a daily basis on their own. Targeting PA may promote homebased therapy for CLD patients. In Japan, guidelines for PA in everyday life are indicated as 23 metabolic equivalents-hour/ week (METs-h/w) for ages 18 - 64 years, and 10 METs-h/w 
for ages 65 years and above [12]. However, there are little data investigating the impact of PA on sarcopenia in patients with CLD. Hand grip strength (HGS) is used in many sarcopenia diagnostic guidelines $[2,3,13]$. HGS is highly correlated with skeletal muscle mass by computed tomography (CT) and can be easily measured [14]. In this study, grip strength, an important item for diagnosis of sarcopenia, was set the main outcome. Therefore, the purpose of this study was to clarify the impact of PA on the prevention of muscle weakness in patients with CLD.

\section{Materials and Methods}

\section{Study population and design}

This was a prospective observational study conducted at the CLD Outpatient Clinics, Saiseikai Niigata Hospital, Niigata, Japan. Ethical approval was obtained from the Ethics Committees of both Niigata University (approval number: 2017-0082) and Saiseikai Niigata Hospital (approval number: E17-06). Participants signed an informed consent before being enrolled into the study. This study was conducted in accordance with the Declaration of Helsinki. Inclusion criteria were outpatients with CLD who were: 1) Twenty years of age or older; 2) Those who were able to measure HGS. Exclusion criteria were as follows: 1) No response to questionnaire; 2) Dementia. This study was conducted in two periods (from September 2017 to November 2017 (baseline), and from January 1, 2018 to March 31, 2018 (endpoint)). Patient characteristics were investigated at baseline. The second HGS measurement was performed at the endpoint.

\section{Patient characteristics}

Patient characteristics were obtained from questionnaires and medical records, namely age, sex, body mass index (BMI), etiology of liver disease, existence of HCC, type of drug therapy, living alone or living together, smoking, drinking, frequency of driving a motor vehicle, employment status, home task, opinions concerning diet, and exercise. Blood samples were obtained from each patient before the computed tomography (CT) examination for biochemical and hematological evaluation to measure the following: serum albumin (Alb), alkaline phosphatase (ALP), alanine transaminase (ALT), aspartate aminotransferase (AST), blood urea nitrogen (BUN), creatine kinase (CK), creatinine (Cre), cholinesterase (ChE), estimated glomerular filtration rate (eGFR), hemoglobin (Hb), hemoglobin A1c (HbA1c), $\alpha$-fetoprotein (AFP), prothrombin time-international normalized ratio (PT-INR), total bilirubin (T-bil), total cholesterol (Tcho), total protein (TP), triglyceride (TG), red blood cell count (RBC), white blood cell count (WBC), total lymphocyte count (TLC), and platelet count (Plt). HbAlc was expressed as a National Glycohemoglobin Standardization Program (NGCP) unit. Malnutrition was evaluated by the controlling nutritional status index score (CONUT-score) [15]. CONUT-score was obtained based on the serum Alb and T-cho levels and TLC. The fibrosis 4 index (Fib-4 index) which evaluates liver fibrosis was scored based on age, ALT, AST, and Plt [16-18]. The albumin-bilirubin
(ALBI) grade, an objective measurement of liver function, was scored based on the Alb and T-bil levels [19, 20]. The definition of sarcopenia in this study followed the guidelines of the Japan Society of Hepatology [13].

\section{Evaluation of PA}

PA was evaluated using the International Physical Activity Questionnaire (usual 7 days, short version) (IPAQ), which comprises four questions: vigorous intensity, moderate intensity, walking, sitting and lying down time in a day [21-23]. PA was estimated by adding the products of reported time for each item by the METs value that was specific to each PA category. In Japan, guidelines for routine PA are indicated as 23 METs$\mathrm{h} / \mathrm{w}$ for individuals aged 18 - 64 years, and $10 \mathrm{METs}-\mathrm{h} / \mathrm{w}$ for those aged 65 years and above [12]. Therefore, it was set as the cut-off point of physical activity (METs-h/w) in this study.

\section{Measurement of HGS}

The HGS was measured using a digital hand dynamometer (TKK 5401 Grip-D, Takei, Tokyo, Japan) with a precision of $0.1 \mathrm{~kg}$. With the patient standing upright, the left and right grip strength was measured twice, respectively, and the higher average was adopted. The first measurement was conducted from September to November 2017, and the second, from January to March 2018.

\section{Statistical analysis}

Participants were divided into four groups: younger (age $<65$ years): PA $<23$ METs-h/w and PA $\geq 23$ METs-h/w; elderly (age $\geq 65$ years): PA $<10 \mathrm{METs}-\mathrm{h} / \mathrm{w}$ and PA $\geq 10 \mathrm{METs}-\mathrm{h} / \mathrm{w}$. Changes in HGS from baseline to endpoint in each group were compared. Continuous variables were expressed as means \pm standard deviation or median (interquartile range) and compared using the Mann-Whitney U test. Categorical variables and nominal variables were expressed in frequency (percentage) and compared using the Fisher's exact test. We performed multivariate analysis using the logistic regression model with age, sex, HCC, BCAAs supplementation, ALBI grade, and PA related to changes in grip strength as variables. Values of $\mathrm{P}<$ 0.05 were considered statistically significant. Statistical analyses were performed using EZR ver. 1.37 (Saitama Medical Center, Jichi Medical University, Saitama, Japan) [24].

\section{Results}

\section{Patient characteristics at baseline}

A total of 214 participants with CLD were enrolled into this study. During the study period, 31 participants were excluded: 17 participants declined to participate, six participants could not measure HGS, four participants dropped out, two par- 


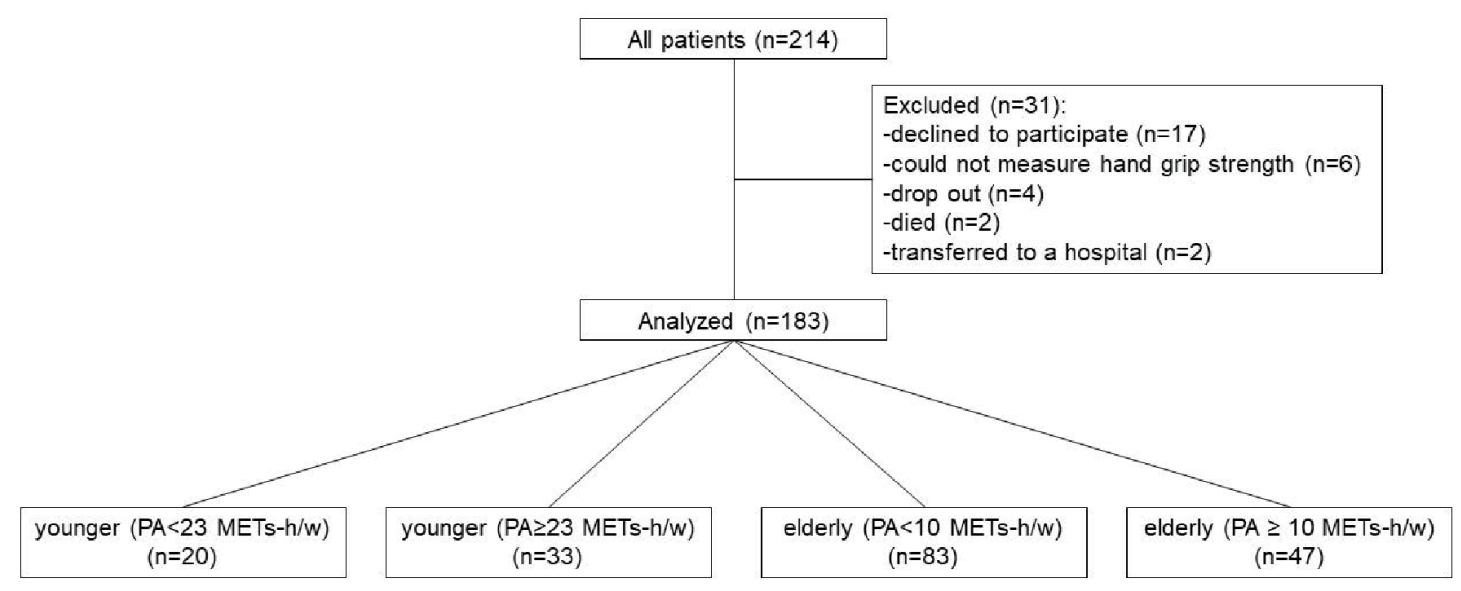

Figure 1. Flowchart indicating selection of participants and grouping according to age and PA. PA: physical activity.

ticipants died, and two participants were transferred to other hospital. Finally, a total of 183 participants were analyzed in the present study (Fig. 1). Among them, 107 participants $(58 \%)$ were men, and the median age was 69.0 years. The most common etiology of liver disease was hepatitis $\mathrm{C}$ in 69 cases $(38 \%)$, and HCC existed in 42 participants (23\%, Table 1$)$.

\section{Comparison of characteristics and biochemical measure- ments in young patients}

The sedentary time of low PA (PA $<23$ METs-h/w) was significantly longer than that of high PA (PA $\geq 23 \mathrm{METs}-\mathrm{h} / \mathrm{w})$ in young patients (Table 2). There were no other significant variables between the two groups. There were no significant differences in the biochemical measurements between the two groups (Table 3 ).

\section{Comparison of changes in HGS between low PA and high PA in young patients}

Changes in HGS in younger patients are shown in Table 4. There were no significant differences in changes of HGS (-0.02 $(-1.83,1.47)$ vs. $0.25(-2.45,2.05), \mathrm{P}=0.956)$.

\section{Comparison of characteristics and biochemical measure- ments in elderly patients}

Comparison of patient characteristics among the elderly is shown in Table 5. Patients with low PA (PA $<10$ METs-h/w) had more sarcopenia $(31.9 \%$ vs. $7.2 \%, \mathrm{P}<0.01)$ than patients with high PA (PA $\geq 10 \mathrm{METs}-\mathrm{h} / \mathrm{w})$. Patients with low PA had lower HGS (22.95 (17.82, 31.00) kg vs. 27.05 (21.80, 33.73) $\mathrm{kg}, \mathrm{P}<0.05)$ than those with high PA. In the elderly, the sedentary time of patients with low PA was significantly longer (7.0 (5.0, 10.0) h/day vs. $5.0(3.0,6.0) \mathrm{h} /$ day, $\mathrm{P}<0.01)$ than those with high PA. In addition, the frequency of living alone was significantly higher in patients with low PA compared with those with high PA $(27.7 \%$ vs. $12.0 \%, \mathrm{P}<0.05)$. The frequency of exercise habit was significantly lower in patients with low PA compared to those with high PA $(55.3 \%$ vs. $88.0 \%, \mathrm{P}<$ $0.01)$. Table 6 shows the comparison of the biochemical meas-

Table 1. Characteristics of Patients With Chronic Liver Disease $(N=183)$

\begin{tabular}{|ll}
\hline Age (years) & $\mathbf{6 9 . 0}(\mathbf{6 3 . 0 , 7 5 . 0 )}$ \\
\hline Male & $107(58 \%)$ \\
\hline Body mass index $\left(\mathrm{kg} / \mathrm{m}^{2}\right)$ & $23.41(20.69,25.23)$ \\
\hline Etiology of liver disease & \\
$\quad$ Hepatitis B & $46(25 \%)$ \\
$\quad$ Hepatitis C & $69(38 \%)$ \\
$\quad$ Alcoholic hepatitis & $22(12 \%)$ \\
$\quad$ Nonalcoholic steatohepatitis & $23(13 \%)$ \\
$\quad$ Autoimmune hepatitis & $1(<1 \%)$ \\
$\quad$ Primary biliary cirrhosis & $2(<1 \%)$ \\
$\quad$ Others & $20(11 \%)$ \\
\hline Hepatocellular carcinoma & $42(23 \%)$ \\
Sarcopenia & $22(12 \%)$ \\
\hline ALBI grade (1/2/3) & $145 / 37 / 1$ \\
Branched-chain amino acids (intake) & $67(37 \%)$ \\
Living alone (yes) & $32(17 \%)$ \\
Currently smoking (yes) & $32(17 \%)$ \\
Currently drinking (yes) & $41(22 \%)$ \\
Driving of cars (usually) & $149(81 \%)$ \\
Employment status (retired) & $94(51 \%)$ \\
\hline Do home tasks (yes) & $100(55 \%)$ \\
Healthy eating (yes) & $157(86 \%)$ \\
Exercise habits (yes) & $133(73 \%)$ \\
Observation period (days) & $119(96.5,140)$ \\
\hline
\end{tabular}

Data were expressed as median (interquartile range) or as $\mathrm{n}(\%)$. ALBI: albumin-bilirubin. 
Table 2. Comparison of Patient's Characteristics in Young Patients at Baseline

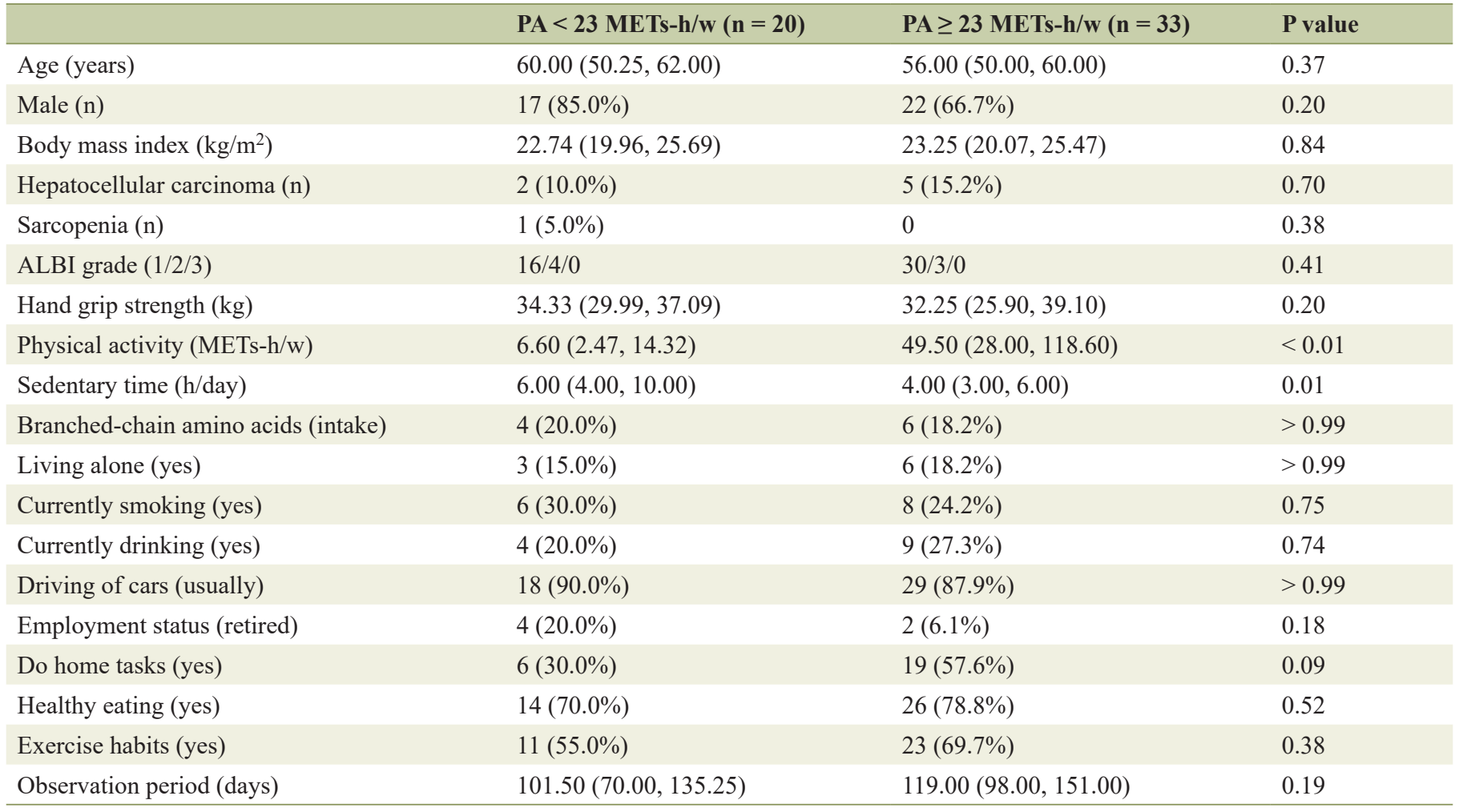

Data were expressed as median (interquartile range), or as $\mathrm{n}(\%)$. Continuous variables were compared by Mann-Whitney $U$ test. Categorical variables were compared by Fisher's exact test. PA: physical activity; METS-h/w: metabolic equivalents-hour/week; ALBI: albumin-bilirubin.

urements in the elderly. The CK of patients with low PA was significantly lower than those with high PA.

\section{Comparison of changes in HGS between low PA and high PA in elderly patients}

Changes of HGS in the elderly are shown in Table 7. Patients with low PA had significantly reduced HGS compared to those with high PA $(-1.00(-2.27,0.55) \mathrm{kg}$ vs. $0.10(-1.40,1.10) \mathrm{kg}$, $\mathrm{P}<0.05)$.

\section{Factors related to prevention of decrease in HGS}

We defined patients whose change in HGS was $<0$ as those with a decrease in HGS. We divided them into decreased HGS (change in HGS $<0$ ) and increased HGS (change in HGS $\geq 0$ ) groups and analyzed the data using logistic regression analysis. Logistic regression analyses identified PA as the independent factor for increased HGS (odds ratio: $1.91,95 \%$ confidence interval: 1.00 - 3.62, $\mathrm{P}=0.049$ ) (Table 8).

\section{Discussion}

Our results showed that PA of $10 \mathrm{METs}-\mathrm{h} / \mathrm{w}$ or more may pre- vent a decrease in HGS in the elderly. To our knowledge, our study is the first report to examine changes in HGS and PA in patients with CLD. In our results, the HGS of low PA in elderly patients decreased by about $1 \mathrm{~kg}$ in around 4 months. No significant effect of PA was observed on HGS change in young patients. A previous study reported that the average HGS worsened by $-0.38 \mathrm{~kg}$ per 3 months in patients with cirrhosis awaiting liver transplantation [25]. This is partially consistent with our results that the HGS of patients with CLD decreases significantly in only a few months. However, the previous study population had poor liver function; $65 \%$ of the study population were Child-Pugh class B [25]. We did not assess liver function with Child-Pugh class, but at least $70 \%$ were ALBI grade 1 , and the liver function was maintained. Our report explained that even though liver function was maintained, the decrease in HGS was caused by low PA. In our study, the HGS of the elderly with low PA was significantly lower than that of the elderly with high PA $(22.95$ (17.82, 31.00) vs. 27.05 $(21.80,33.73), \mathrm{P}<0.01)$ at baseline. This is consistent with previous reports that have shown that female volunteers over 60 years of age who did not meet the PA recommendations had low physical function [26]. Our finding that the HGS of elderly patients with CLD and low PA decreased significantly indicated that decrease in physical function and PA affect each other. Similar results were reported by Laddu et al [27]. In their study, the high PA groups $(>1,200 \mathrm{METs}-\mathrm{min} / \mathrm{w})$ had stronger grip strength than sedentary women $(<100 \mathrm{METs}-\mathrm{min} / \mathrm{w})$. 
Table 3. Comparison of Biochemical Measurements in Young Patients at Baseline

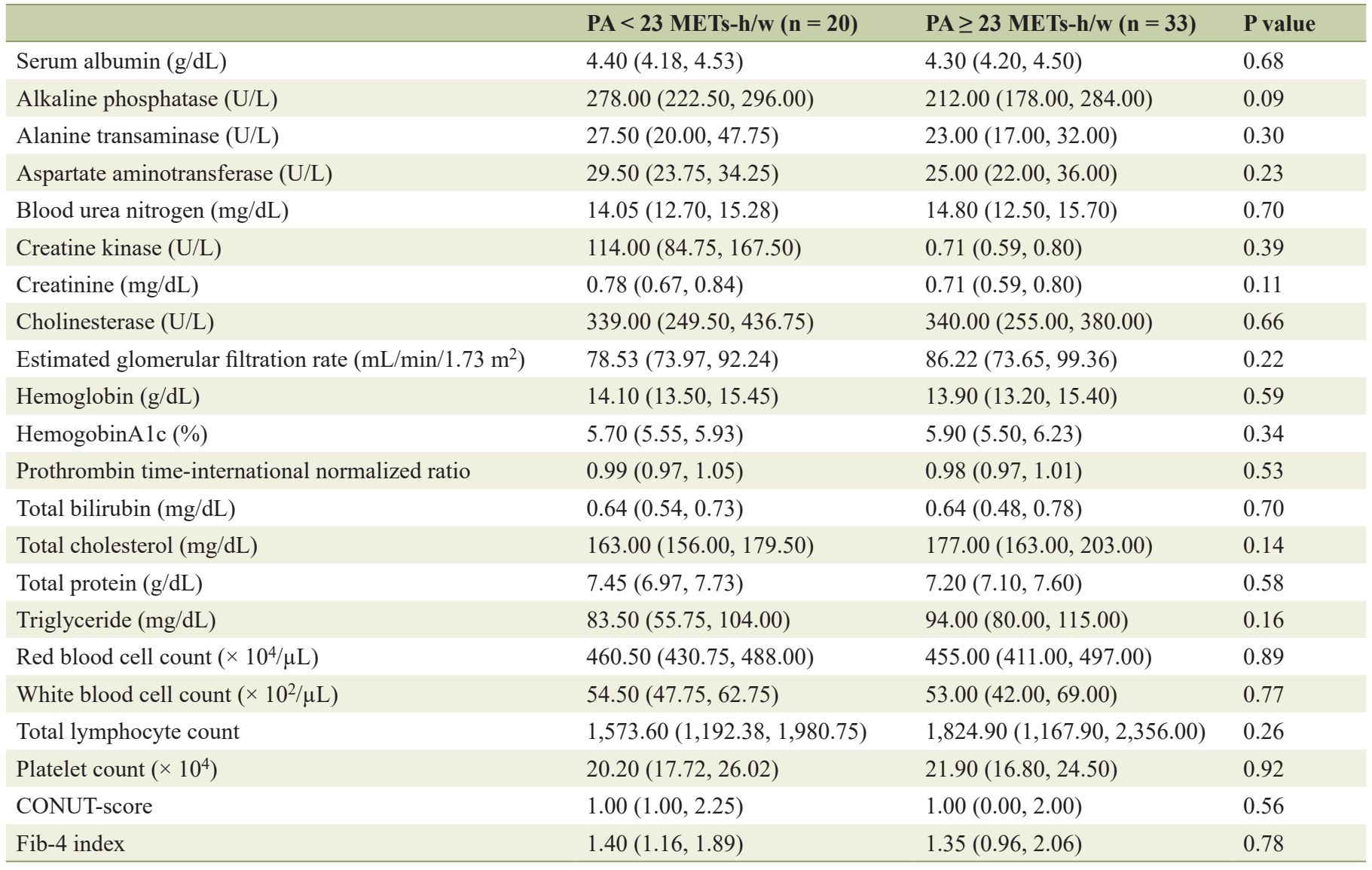

Data were expressed as median (interquartile range). Continuous variables were compared by the Mann-Whitney U test. PA: physical activity; METSh/w: metabolic equivalents-hour/week; CONUT-score: controlling nutritional status index score; Fib-4 index: fibrosis 4 index.

Furthermore, it was reported that maintaining high PA levels over time was associated with better lower extremity function [27]. Although different from the cut-off value of PA in our study, high PA was important for maintaining physical function, which is also true for patients with CLD. The cut-off value of PA in our study is recommended by the Ministry of Health, Labour and Welfare in Japan, so this cut-off value is appropriate for the Japanese population. We examined the reason for the absence of a significant change in young patients. In this study, the total PA was calculated, but the intensity of activity was not considered. The World Health Organization (WHO) recommends that adults aged 18 - 64 years should perform at least $150 \mathrm{~min}$ of moderate-intensity aerobic PA throughout the week or engage in at least 75 min of vigorous-intensity aerobic PA throughout the week or an equivalent combination of mod- erate- and vigorous-intensity activity [28]. Therefore, a future study is needed to consider the effect of exercise intensity on muscle strength.

The low PA group showed a longer sedentary time than the high PA group in both young and elderly patients in our results. The sedentary time of low PA in elderly patients was $7 \mathrm{~h}$ /day. The sedentary time of general elderly people in Japan was about $420 \mathrm{~min}(=7 \mathrm{~h}) /$ day [29], which was the same as the low PA in our study. Therefore, patients with CLD also had sedentary characteristics comparable to those of the general elderly population in Japan. Our study findings showed that low HGS and low PA and long sedentary time were identical in the elderly patients with low PA. The significant differences in each variable observed in our study were partially in agreement with the findings of previous studies. A previous

Table 4. Comparison of Changes in Hand Grip Strength From the Baseline to the Endpoint in Young Patients

\begin{tabular}{llll} 
& PA $<23$ METs-h/w $(\mathbf{n}=\mathbf{2 0})$ & PA $\geq 23$ METs-h/w $(\mathbf{n}=\mathbf{3 3})$ & P value \\
\hline Changes in hand grip strength $(\mathrm{kg})$ & $-0.02(-1.83,1.47)$ & $0.25(-2.45,2.05)$ & 0.96 \\
Rate of change $(\%)$ & $-0.06(-5.85,4.43)$ & $0.69(-6.76,6.39)$ & 0.99 \\
\hline
\end{tabular}

Data were expressed as median (interquartile range). Variables were compared by the Mann-Whitney U test. PA: physical activity; METS-h/w: metabolic equivalents-hour/week. 
Table 5. Comparison of Patient's Characteristics in Elder Patients at Baseline

\begin{tabular}{|c|c|c|c|}
\hline & PA $<10$ METs-h/w $(n=47)$ & $P A \geq 10$ METs-h/w $(n=83)$ & $P$ value \\
\hline Age (years) & $73.00(68.50,78.00)$ & $71.00(68.00,77.00)$ & 0.32 \\
\hline Male (n) & $22(46.8 \%)$ & $46(55.4 \%)$ & 0.37 \\
\hline Hepatocellular carcinoma (n) & $14(29.8 \%)$ & $21(25.3 \%)$ & 0.68 \\
\hline Sarcopenia (n) & $15(31.9 \%)$ & $6(7.2 \%)$ & $<0.01$ \\
\hline Hand grip strength (kg) & $22.95(17.82,31.00)$ & $27.05(21.80,33.73)$ & $<0.01$ \\
\hline Physical activity (METS-h/w) & $4.40(1.65,6.60)$ & $25.10(15.62,49.70)$ & $<0.01$ \\
\hline Sedentary time (h/day) & $7.00(5.00,10.00)$ & $5.00(3.00,6.00)$ & $<0.01$ \\
\hline Branched-chain amino acids (intake) & $19(40.4 \%)$ & $38(45.8 \%)$ & 0.59 \\
\hline Living alone (yes) & $13(27.7 \%)$ & $10(12.0 \%)$ & 0.03 \\
\hline Employment status (retired) & $37(78.7 \%)$ & $51(61.4 \%)$ & 0.05 \\
\hline Do home tasks (yes) & $30(63.8 \%)$ & $45(54.2 \%)$ & 0.36 \\
\hline Healthy eating (yes) & $42(89.4 \%)$ & $75(90.4 \%)$ & $>0.99$ \\
\hline Exercise habits (yes) & $26(55.3 \%)$ & $73(88.0 \%)$ & $<0.01$ \\
\hline Observation period (days) & $126.00(105.00,147.00)$ & $112.00(91.00,140.00)$ & 0.05 \\
\hline
\end{tabular}

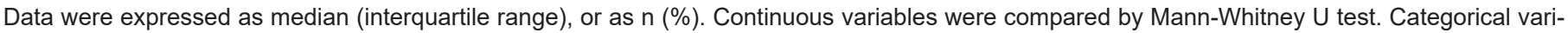
ables were compared by Fisher's exact test. PA: physical activity; METS-h/w: metabolic equivalents-hour/week; ALBI: albumin-bilirubin.

study suggested that sedentary time was related to mortality and obesity in people over the age of 50 years independent of PA levels [30, 31]. Moreover, low PA and a long sedentary time in elderly people have been reported to be associated with high obesity rates and low $\operatorname{HGS}[32,33]$. However, in our study, BMI was not significantly different between elderly patients with low PA and high PA, which is inconsistent with the findings of previous reports. This result may suggest that loss of physical function or loss of PA may occur before weight loss. Our study showed that characteristics of low PA included more living alone and more sarcopenia than those of high PA in elderly patients. A previous study showed that the quality of life domain of social relationships is declining in the low PA population [26]. Our results partially explained the reality of the decline in the quality of life domain of social relationships. In another previous study in Japan, it was found that community-dwelling women with sarcopenia lived with their children and/or grandchildren more than those without sarcopenia, who lived alone [34]. Living alone requires adequate physical function. However, it is reported that the elderly who live and eat alone have a significantly lower food diversity and food intake frequency than those who eat together their family often [35], and have lower food accessibility, related to shopping difficulty [36]. Therefore, even if the elderly living alone have independent lives, there is a concern that the quality of their food intake may deteriorate their nutritional status. Although the difference of CONUT-score was not significant between the elderly patients with low PA and high PA, the elderly patients with low PA, who had a high frequency of living alone, had a CONUT-score of 2 points in our study. It showed mild malnutrition and may be associated with poor PA and decreased HGS.

Our results have shown that PA of $10 \mathrm{METs}-\mathrm{h} / \mathrm{w}$ or more may prevent a decrease in HGS in elderly patients with CLD. The current study outcome (PA $\geq 10 \mathrm{METs}-\mathrm{h} / \mathrm{w}$ for the elderly) may be the target of home-based therapy for patients with CLD. Previous studies have reported that muscle mass, muscular strength and physical function are improved by intervention combined with exercise and nutrition in patients with cirrhosis [11]. These interventions differ from our observational study in that exercise programs were performed by experts. To date, there are reports on home-based therapy that state that HGS, leg strength, and muscle mass increased after 3 months of exercise therapy in cirrhotic patients, including due to 2,000 steps per day and BCAA supplementation [37]. Additionally, patients with LC of less than 5,000 steps per day have been reported to have a high prevalence of sarcopenia, and sufficient PA may prevent sarcopenia [38]. However, attention should also be paid to routine PA (for example: household chores, gardening, pet care) other than walking. Combining these PAs and building a sustainable individual PA program will lead to the prevention of sarcopenia in older patients with CLD.

Our study has three limitations. First, this study was performed in a single institution, and a validation study in multiple institutions is needed in the future. Second, as the etiology is not limited, lifestyles may differ depending on the cause of 
Table 6. Comparison of Biochemical Measurements in Elder Patients at Baseline

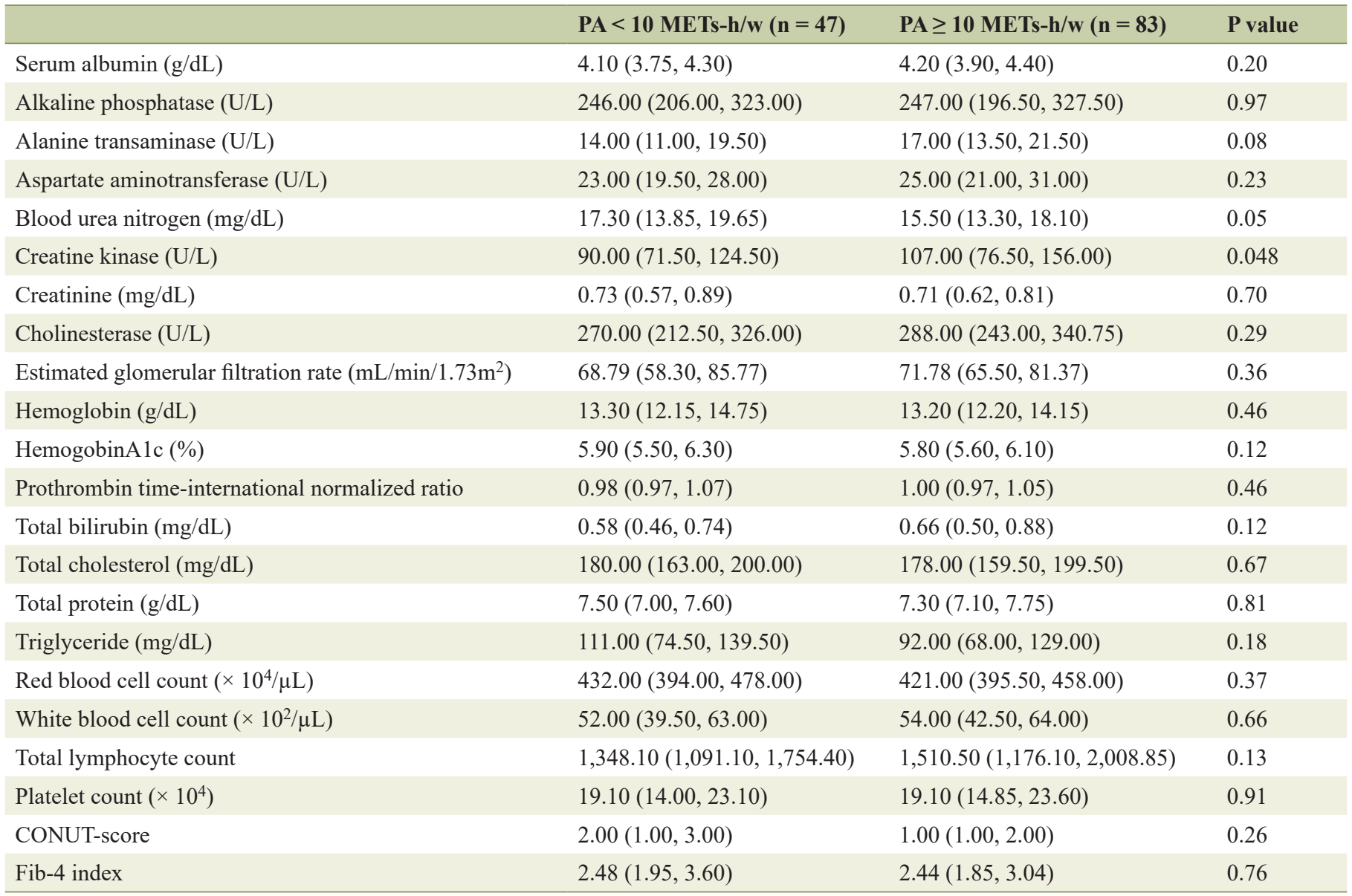

Data were expressed as median (interquartile range). Continuous variables were compared by Mann-Whitney U test. CONUT-score: controlling nutritional status index score; Fib-4 index: fibrosis 4 index. PA: physical activity; METS-h/w: metabolic equivalents-hour/week.

Table 7. Comparison of Changes in Hand Grip Strength From Baseline to Endpoint in Elder Patients

\begin{tabular}{llll} 
& Low PA $(\mathbf{n}=\mathbf{4 7})$ & High PA $(\mathbf{n}=\mathbf{8 3})$ & P value \\
\hline Changes in hand grip strength $(\mathrm{kg})$ & $-1.00(-2.27,0.55)$ & $0.10(-1.40,1.10)$ & 0.02 \\
Rate of change (\%) & $-5.24(-8.59,2.94)$ & $0.44(-4.17,4.66)$ & 0.03 \\
\hline
\end{tabular}

Data were expressed as median (interquartile range). Variables were compared by Mann-Whitney U test. PA: physical activity.

Table 8. Logistic Regression Analysis of Variables Associated With Prevention of Decreased HGS

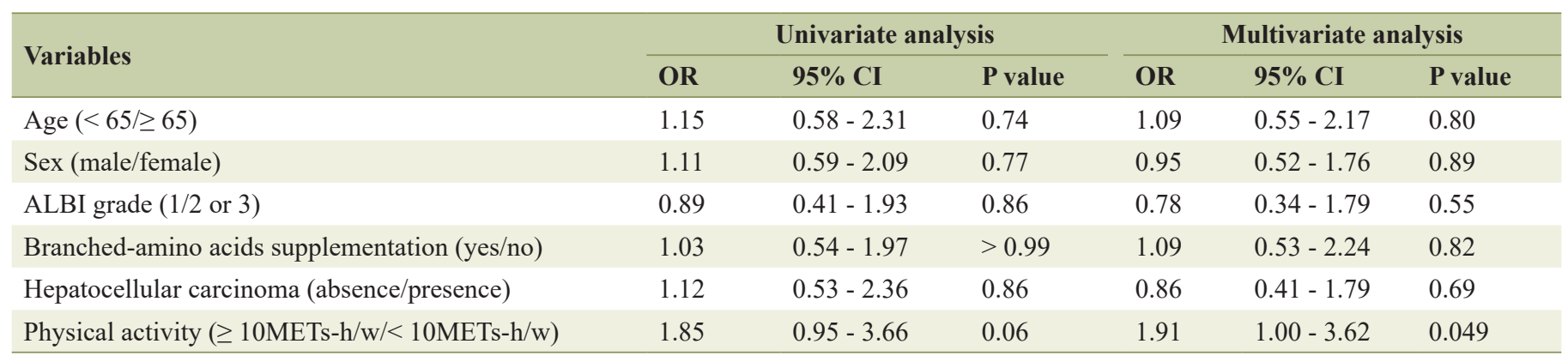

Data were analyzed by logistic regression analysis model. HGS: hand grip strength; OR: odds ratio; CI: confidence interval; ALBI: albumin-bilirubin; METs: metabolic equivalents-hour/week. 
the etiology. In particular, alcoholic hepatitis and nonalcoholic steatohepatitis are greatly affected by lifestyle habits. Finally, the amount of PA used in this study was based on a questionnaire and there might be a possibility that the accuracy was lower than the PA amount measured by triaxial accelerometers. Accurate amount of PA measurement by a wearable devise will lead to developments in exercise therapy in the future. A randomized controlled trial, with PA managed using triaxial accelerometers, is needed to clarify the effect of PA on muscle strength in patients with CLD.

In conclusion, young patients with low PA had the characteristics of a long sedentary time; however, there was no loss of HGS. Elderly patients with CLD and low PA had significantly reduced HGS compared with elderly patients with CLD and high PA. Therefore, for elderly patients with CLD, PA above $10 \mathrm{METs}-\mathrm{h} / \mathrm{w}$ may prevent sarcopenia.

\section{Acknowledgments}

The authors thank Shiori Hirosawa and Miyu Munakata for date collection.

\section{Financial Disclosure}

This work was supported financially by Niigata University Graduate School of Health Sciences.

\section{Conflict of Interest}

All authors have no conflict of interest to declare.

\section{Informed Consent}

Informed consents from the participants were obtained.

\section{Author Contributions}

$\mathrm{KO}$, TI and Yu Koyama: concept and design of the study; KO, $\mathrm{MS}, \mathrm{HN}$ and $\mathrm{HH}$ : date acquisition; $\mathrm{AH}$ and $\mathrm{TH}$ evaluated $\mathrm{CT}$ examinations; KO analyzed the data and drafted the manuscript; YM, EY, HA and Yuko Kikuchi critically reviewed the manuscript.

\section{References}

1. Rosenberg I. Summary comments: epidemiological and methodological problems in determining nutritional status of older persons. Am J Clin Nutr. 1989;50:1231-1233.

2. Cruz-Jentoft AJ, Baeyens JP, Bauer JM, Boirie Y, Cederholm T, Landi F, Martin FC, et al. Sarcopenia: European consensus on definition and diagnosis: Report of the European Working Group on Sarcopenia in Older People.
Age Ageing. 2010;39(4):412-423.

3. Chen LK, Liu LK, Woo J, Assantachai P, Auyeung TW, Bahyah KS, Chou MY, et al. Sarcopenia in Asia: consensus report of the Asian Working Group for Sarcopenia. J Am Med Dir Assoc. 2014;15(2):95-101.

4. Hanai T, Shiraki M, Nishimura K, Ohnishi S, Imai K, Suetsugu A, Takai K, et al. Sarcopenia impairs prognosis of patients with liver cirrhosis. Nutrition. 2015;31(1):193199.

5. Hanai T, Shiraki M, Watanabe S, Kochi T, Imai K, Suetsugu A, Takai K, et al. Sarcopenia predicts minimal hepatic encephalopathy in patients with liver cirrhosis. Hepatol Res. 2017;47(13):1359-1367.

6. Fujiwara N, Nakagawa H, Kudo Y, Tateishi R, Taguri M, Watadani T, Nakagomi R, et al. Sarcopenia, intramuscular fat deposition, and visceral adiposity independently predict the outcomes of hepatocellular carcinoma. J Hepatol. 2015;63(1):131-140.

7. Kamachi S, Mizuta T, Otsuka T, Nakashita S, Ide Y, Miyoshi A, Kitahara K, et al. Sarcopenia is a risk factor for the recurrence of hepatocellular carcinoma after curative treatment. Hepatol Res. 2016;46(2):201-208.

8. Harimoto N, Shirabe K, Yamashita YI, Ikegami T, Yoshizumi T, Soejima Y, Ikeda T, et al. Sarcopenia as a predictor of prognosis in patients following hepatectomy for hepatocellular carcinoma. Br J Surg. 2013;100(11):15231530.

9. Morley JE, Argiles JM, Evans WJ, Bhasin S, Cella D, Deutz NE, Doehner W, et al. Nutritional recommendations for the management of sarcopenia. J Am Med Dir Assoc. 2010;11(6):391-396.

10. Denison HJ, Cooper C, Sayer AA, Robinson SM. Prevention and optimal management of sarcopenia: a review of combined exercise and nutrition interventions to improve muscle outcomes in older people. Clin Interv Aging. 2015;10:859-869.

11. Tandon P, Ismond KP, Riess K, Duarte-Rojo A, Al-Judaibi B, Dunn MA, Holman J, et al. Exercise in cirrhosis: Translating evidence and experience to practice. J Hepatol. 2018;69(5):1164-1177.

12. Miyachi M. Measures of physical activity and exercise for health promotion by the Ministry of Health, Labour and Welfare. J Phys Fit Sports Med. 2012;1:467-472.

13. Nishikawa H, Shiraki M, Hiramatsu A, Moriya K, Hino K, Nishiguchi S. Japan Society of Hepatology guidelines for sarcopenia in liver disease (1st edition): Recommendation from the working group for creation of sarcopenia assessment criteria. Hepatol Res. 2016;46(10):951-963.

14. Itoh S, Shirabe K, Yoshizumi T, Takeishi K, Harimoto N, Ikegami T, Kawanaka H, et al. Skeletal muscle mass assessed by computed tomography correlates to muscle strength and physical performance at a liver-related hospital experience. Hepatol Res. 2016;46(4):292-297.

15. Ignacio de Ulibarri J, Gonzalez-Madrono A, de Villar NG, Gonzalez P, Gonzalez B, Mancha A, Rodriguez F, et al. CONUT: a tool for controlling nutritional status. First validation in a hospital population. Nutr Hosp. 2005;20(1):38-45.

16. Vallet-Pichard A, Mallet V, Nalpas B, Verkarre V, Nalpas 
A, Dhalluin-Venier V, Fontaine H, et al. FIB-4: an inexpensive and accurate marker of fibrosis in HCV infection. comparison with liver biopsy and fibrotest. Hepatology. 2007;46(1):32-36.

17. Sumida Y, Yoneda M, Hyogo H, Itoh Y, Ono M, Fujii H, Eguchi Y, et al. Validation of the FIB4 index in a Japanese nonalcoholic fatty liver disease population. BMC Gastroenterol. 2012;12:2.

18. Sterling RK, Lissen E, Clumeck N, Sola R, Correa MC, Montaner J, M SS, et al. Development of a simple noninvasive index to predict significant fibrosis in patients with HIV/HCV coinfection. Hepatology. 2006;43(6):13171325.

19. Johnson PJ, Berhane S, Kagebayashi C, Satomura S, Teng M, Reeves HL, O'Beirne J, et al. Assessment of liver function in patients with hepatocellular carcinoma: a new evidence-based approach-the ALBI grade. J Clin Oncol. 2015;33(6):550-558.

20. Hiraoka A, Kumada T, Kudo M, Hirooka M, Tsuji K, Itobayashi E, Kariyama K, et al. Albumin-Bilirubin (ALBI) grade as part of the evidence-based clinical practice guideline for HCC of the Japan society of hepatology: a comparison with the liver damage and child-pugh classifications. Liver Cancer. 2017;6(3):204-215.

21. Craig CL, Marshall AL, Sjostrom M, Bauman AE, Booth ML, Ainsworth BE, Pratt M, et al. International physical activity questionnaire: 12 -country reliability and validity. Med Sci Sports Exerc. 2003;35(8):1381-1395.

22. Tomioka K, Iwamoto J, Saeki K, Okamoto N. Reliability and validity of the International Physical Activity Questionnaire (IPAQ) in elderly adults: the Fujiwara-kyo Study. J Epidemiol. 2011;21(6):459-465.

23. Murase N, Katsumura T, Ueda C, Inoue S, Shimomitsu $\mathrm{T}$. Validity and reliability of Japanese version of international physical activity questionnaire. J Heal Welf Stat. 2002;49(11):1-9.

24. Kanda Y. Investigation of the freely available easy-touse software 'EZR' for medical statistics. Bone Marrow Transplant. 2013;48(3):452-458.

25. Lai JC, Dodge JL, Sen S, Covinsky K, Feng S. Functional decline in patients with cirrhosis awaiting liver transplantation: Results from the functional assessment in liver transplantation (FrAILT) study. Hepatology. 2016;63(2):574-580.

26. Nawrocka A, Polechonski J, Garbaciak W, Mynarski W. Functional fitness and quality of life among women over 60 years of age depending on their level of objectively measured physical activity. Int J Environ Res Public Health. 2019;16(6):972.

27. Laddu DR, Wertheim BC, Garcia DO, Brunner R, Groessl E, Shadyab AH, Going SB, et al. Associations between self-reported physical activity and physical performance measures over time in postmenopausal women: the women's health initiative. J Am Geriatr Soc. 2017;65(10):2176-2181.

28. World Health Organization (WHO). Global Recommendations on Physical Activity for Health. Geneva, 2010.

29. Chen T, Kishimoto H, Honda T, Hata J, Yoshida D, Mukai N, Shibata M, et al. Patterns and Levels of Sedentary Behavior and Physical Activity in a General Japanese Population: The Hisayama Study. J Epidemiol. 2018;28(5):260-265.

30. de Rezende LF, Rey-Lopez JP, Matsudo VK, do Carmo Luiz O. Sedentary behavior and health outcomes among older adults: a systematic review. BMC Public Health. 2014;14:333.

31. Rosenberg D, Cook A, Gell N, Lozano P, Grothaus L, Arterburn D. Relationships between sitting time and health indicators, costs, and utilization in older adults. Prev Med Rep. 2015;2:247-249.

32. Bann D, Hire D, Manini T, Cooper R, Botoseneanu A, McDermott MM, Pahor M, et al. Light Intensity physical activity and sedentary behavior in relation to body mass index and grip strength in older adults: crosssectional findings from the Lifestyle Interventions and Independence for Elders (LIFE) study. PLoS One. 2015;10(2):e0116058.

33. Dodds R, Kuh D, Aihie Sayer A, Cooper R. Physical activity levels across adult life and grip strength in early old age: updating findings from a British birth cohort. Age Ageing. 2013;42(6):794-798.

34. Momoki C, Habu D, Ogura J, Tada A, Hasei A, Sakurai K, Watanabe H. Relationships between sarcopenia and household status and locomotive syndrome in a community-dwelling elderly women in Japan. Geriatr Gerontol Int. 2017;17(1):54-60.

35. Ishikawa M, Takemi Y, Yokoyama T, Kusama K, Fukuda Y, Nakaya T, Nozue M, et al. "Eating Together" is associated with food behaviors and demographic factors of older Japanese people who live alone. J Nutr Health Aging. 2017;21(6):662-672.

36. Ishikawa M, Yokoyama T, Nakaya T, Fukuda Y, Takemi Y, Kusama K, Yoshiike N, et al. Food accessibility and perceptions of shopping difficulty among elderly people living alone in Japan. J Nutr Health Aging. 2016;20(9):904911.

37. Hiraoka A, Michitaka K, Kiguchi D, Izumoto H, Ueki H, Kaneto M, Kitahata S, et al. Efficacy of branchedchain amino acid supplementation and walking exercise for preventing sarcopenia in patients with liver cirrhosis. Eur J Gastroenterol Hepatol. 2017;29(12):1416-1423.

38. Hayashi F, Matsumoto Y, Momoki C, Yuikawa M, Okada G, Hamakawa E, Kawamura E, et al. Physical inactivity and insufficient dietary intake are associated with the frequency of sarcopenia in patients with compensated viral liver cirrhosis. Hepatol Res. 2013;43(12):1264-1275. 